\title{
Evaluation of pancreatic lipase and gamma-glutamyl transferase in acute pancreatitis
}

\section{Abstract}

Background and objective: Pancreas is a large organ behind the stomach that produces digestive enzymes and a number of hormones. Pancreatitis is a condition characterized by inflammation of the pancreas. When the digestive enzymes are activated before they are released into the small intestine, they begin attacking the pancreas, and pancreatic damage happens. This study aimed to evaluate the association between pancreatic lipase, gamma-glutamyl transferase, and acute pancreatitis in patients living in Erbil city.

Methods: The present study was carried out at the College of Medicine, Hawler Medical University from December 2017 to July 2018. A total of 35 patients with acute pancreatitis and 25 healthy controls were investigated. Examinations of serum pancreatic lipase and gamma glutamyl transferase were carried out spectrophotometrically for both groups using commercial diagnostics kits.

Results: The results showed that the mean level of serum pancreatic lipase in patients with pancreatitis was significantly higher $(P<0.01)$ compared to normal healthy control. The mean serum gamma glutamyl transferase was higher in patients with acute pancreatitis when compared to the reference group but not statistically significant.

Conclusion: In the frame of this work, we delineate that pancreatic diseases had a significant effect on pancreatic enzymes reflected by a significant increase in serum lipase, whereas the level of gamma glutamyl transferase increased marginally.

Keywords: Pancreatitis; Pancreatic lipoprotein lipase; Gamma glutamyl transferase.

\section{Introduction}

Acute pancreatitis is a disease that could result in multiorgan failure, systemic inflammatory response, sepsis, and death. The incidence of acute pancreatitis in the United Kingdom has been estimated to be 100 to 250 per million populations per year. ${ }^{1,2}$ Acute pancreatitis prognostic systems have been refined and developed for many years to assess the severity of the disease in an early stage. Biochemical markers are a common assessment category, which is universally used. The assessment of the severity of acute pancreatitis is not difficult, but assessing the severity of this disease has caused many problems. Knowing methods of early assessment of acute pancreatitis is important. Despite recent advances in gastroenterology, acute pancreatitis is still associated with substantial mortality and morbidity. ${ }^{3,4}$ It is still not possible to manage acute pancreatitis causally, but after about five decades, the diagnosis of acute pancreatitis has significantly improved. Severe acute pancreatitis develops in approximately $20 \%$ of patients at high risk of pancreatic necrosis, leading to systemic complications with mortality rates up to $30 \% .^{5}$ Appropriate and early diagnosis, assessing the severity of the disease, and preventing further attacks of acute pancreatitis are important elements in further therapeutic management. Lipase is characterized by high sensitivity and specificity. The increased serum concentration of lipase in acute pancreatitis is seen within the first 4-8 hours from the

${ }^{1}$ Department of Surgery, College of Medicine, Hawler Medical University, Erbil, I raq.

* Correspondence: aababan@yahoo.com 
onset of symptoms. The peak values usually appear after 24 hours while its concentration remains increased for 7-14 days. However, increased lipase levels are not correlated with the severity of acute pancreatitis. $^{6}$ Lipase, whose half-life in serum (7-13 hours) is longer than that of amylase (2 hours), also has higher sensitivity and specificity than amylase. ${ }^{7}$ Increased Serum lipase concentrations are more specific for acute pancreatitis than increased amylase concentrations. Most studies have reported sensitivities ranging between $55 \%$ and $100 \%$, with specificities above $95 \% .^{8}$ Although there is no optimal diagnostic test for pancreatitis, lipase is preferred in routine clinical practice. ${ }^{9}$ Gamma-glutamyl transferase is an enzyme, which is found in many organs throughout the body. The highest concentrations are found in the liver. It is also elevated in the blood in most diseases that cause liver or bile duct damage. Gamma glutamyl transferase and lipase concentrations do not play a role in assessing the severity of the disease or predicting the prognosis or complications in the case of already diagnosed acute pancreatitis. Both lipase and gamma glutamyl transferase measurements are available and can be easily obtained in hospital practice. This study aimed to find out the possible relationship between serum pancreatic lipase and gammaglutamyl transferase and their usefulness as biochemical parameters in the diagnosis of acute pancreatitis.

\section{Methods}

\section{Subjects}

The present work is a cross-sectional study with a comparison group, carried out from
December 2017 to July 2018 on 35 patients with acute pancreatitis and 25 healthy controls as a collaborative study between the Department of Surgery in the College of Medicine, Hawler Medical University and the Surgery Department in Rizgary Teaching Hospital in Erbil city. The subjects of our study were grouped into patients with acute pancreatitis, a condition characterized by inflammation of the pancreas, and healthy controls. Acute pancreatitis cases have been diagnosed by clinical findings and after doing other blood tests and radiological imaging like ultrasonography and CT scan. The volunteers had no evidence of any disease. The host information of the patients and normal subjects are summarized in Table 1.

\section{Collection of Samples}

Blood samples were collected by vein puncture from patients with pancreatitis who were admitted to Rizgary Teaching Hospital as well as from the control group. The collected blood allowed standing at room temperature until it has clotted. The sample was centrifuged at $3000 \mathrm{rpm}$ for 20 minutes for the removal of any suspended cells. The separated serum was used for the measurement of pancreatic lipase and gamma glutamyl transferase.

\section{Methods}

\section{Estimation of Serum pancreatic lipase}

Serum pancreatic lipase for both groups was determined colorimetrically using BIOLABO SA (Mizy, France) kit. Lipase acts on 1,2- diglycerid to form 2-monoglycerid, which is then hydrolyzed in glycerol and free fatty acids by mono glycerid lipase. Glycerol kinase acts on glucerol to liberate glycerol-3phosphate, which in turn acted on by

Table 1: Information of Patients with pancreatitis and control groups.

\begin{tabular}{lccc}
\hline Groups & Number of subjects & \multicolumn{2}{c}{ Age (years) } \\
& & Mean & Range \\
\hline Control group & 25 & 38.81 & $19-70$ \\
Patients with pancreatic disease & 35 & 40.33 & $19-75$ \\
\hline
\end{tabular}


glycerol-3-phosphate oxidase to generate hydrogen peroxide and subsequently to quinoneimine dye. The rate of formation of quinoneimine dye is directly proportional to the lipase activity in the specimen.

\section{Estimation of serum Gamma glutamyl} transferase

Ready-made kit BIOLABO reagents-mazy France was employed for estimation of serum gamma glutamyl transferase according to Szasz and Tarlow method. After reaction of L-G-glutamyl-P-nitroaniline with glycerine in the presence of gamma glutamyl transferase produce of L-Gglutamyl-glycylglycine and p-nitroaniline. The rate of formation of nitroaniline is directly proportional to gamma glutamyl transferase activity in the specimen.

\section{Statistical analysis:}

Data were evaluated by the statistical package for the social sciences software (SPSS Inc. Chicago, IL, USA). Values are reported as mean $\pm S E$. T-test for independent variables was employed to find out the statistical significance. The levels of serum pancreatic lipase and gamma glutamyl transferase of normal healthy subjects were compared with that of patients with acute pancreatitis. Concerning $P$ value, statistical significance was set at $P<0.05$.

\section{Ethical considerations}

The study was approved by the Research Ethical Committee of Hawler Medical University, College of Medicine, Erbil. An informed and written consent was obtained from each participant before the collection of samples.

\section{Results}

\section{Group I (Control Group):}

The results of the present study are presented in Table 2. The mean \pm S.E value for serum pancreatic lipase was $39 \pm 5.5$ IU/ $L$ with a range of9-61IU/L, the mean \pm S.E value of serum gamma glutamyl transferase was $17 \pm 0.5 \mathrm{IU} / \mathrm{L}$ with a range of 3-29IU/L.

Group II (Patients with pancreatitis):

The mean $\pm S$. E for serum pancreatic lipase was $133 \pm 3.7 \mathrm{IU} / \mathrm{L}$ with a range of $30-210$ $\mathrm{IU} / \mathrm{L}$. The mean value for serum gamma glutamyl transferase was $18.1 \pm 1.1 \mathrm{IU} / \mathrm{L}$ with a range of 4-33 IU.L, as shown in Table 2. The comparison between group I (Control group) and group II (patients with acute pancreatitis) shows significant increases in the level of serum pancreatic lipase (Table 2). Serum gamma glutamyl transferase was higher in patients with acute pancreatitis when compared to the control group, but did not show a statistically significant difference (Table 2).

\section{Discussion}

Acute pancreatitis diagnosis is based usually on the presence of abdominal pain and elevated levels of serum enzymes like amylase and/or lipase. In more than $80 \%$ of patients, acute pancreatitis is mild and resolves without serious morbidity, but in up to $20 \%$, it can be severe and complicated by major morbidity and

Table 2: Biochemical parameters of the studied groups.

\begin{tabular}{lccccc}
\hline Parameters & \multicolumn{2}{c}{ Group I } & \multicolumn{2}{c}{ Group II } & P value \\
IU/L & Control group & Patients with pancreatitis & \\
& Mean \pm S.E & Range & Mean \pm S.E & Range & \\
\hline Pancreatic lipase & $39 \pm 5.5$ & $9-61$ & $133 . \pm 3.7$ & $30-210$ & 0.009 \\
Gamma glutamyl transferase & $17 \pm 0.5$ & $3-29$ & $18.1 \pm 1.1$ & $4-33$ & 0.064 \\
\hline
\end{tabular}

$P$ statistics obtained by student-test. $P<0.05$ indicates a statistically significant difference.

S.E. Standard Error 
mortality. ${ }^{10}$ Many health care centers use serum lipase, amylase, or both to diagnose acute pancreatitis without considering which enzyme may provide better diagnostic accuracy. Lipase has now replaced amylase as the biochemical test of choice in acute pancreatitis. ${ }^{11}$ The levels of these enzymes do not correlate with the severity of the disease. ${ }^{12}$ There is some variability in the reference ranges for lipase, and debate about the optimal value that should be used to diagnose acute pancreatitis. Elevation of serum lipase activity is included in the diagnostic criteria for acute pancreatitis. ${ }^{13}$ Most guidelines recommend 2-3 times the upper limit of normal as the most appropriate cut-off optimal value. ${ }^{14}$ There are a number of other conditions that can elevate lipase, including pancreatic disease, cholecystitis, renal impairment, intestinal ischemia, and malignancy. However, the test's specificity has been shown to be higher than amylase in several studies. ${ }^{15}$ Depending on the cut-offs, specificity could be higher than $95 \%$. For many years, there has been a search for a set of biochemical parameters, which may facilitate rapid assessment of the severity of acute pancreatitis. Due to availability and its low price, lipase is characterized by high specificity and sensitivity. The test sensitivity is affected by the time of the testing and the underlying cause of pancreatitis. Serum amylase was the primary diagnostic marker, but now serum lipase is the preferred test. In this study, we tried to find out the biochemical predictive factors of acute pancreatitis to determine the sequence of the diagnostic method for time and cost-effective purposes. In the present study, serum gamma glutamyl transferase and lipase levels were taken as diagnostic markers of acute pancreatitis. Both gamma glutamyl transferase and lipase are increased in acute pancreatitis, but only serum lipase will be increased in the early stages of acute pancreatitis. Therefore, gamma glutamyl transferase can be used as a follow up to an elevated lipase.
The diagnostic accuracy of lipase appears to be better than that of gamma glutamyl transferase. The latter is not recommended for routine use by itself. However, it can be useful in conjunction with other tests. In this study, gamma glutamyl transferase activity was increased in acute pancreatitis. However, this increase is not specific or diagnostic for pancreatitis because this elevation in an alcoholic patient may be due to hepatic damage. This fact is also confirmed in this study. The data in Table 2 made it readily apparent that serum lipase was significantly increased in acute pancreatitis patients and could be considered a diagnostic marker. In contrast, the elevation in serum gamma glutamyl transferase was not significant. The recent UK guidelines for the management of pancreatitis state: "Where lipase is available it is preferred for the diagnosis of acute pancreatitis," ${ }^{16}$ and according to the British Society of Gastroenterology guidelines for the management of acute pancreatitis, lipase is the main focus for the diagnosis of acute pancreatitis. ${ }^{17}$ A limitation of the study is the lack of information about alcohol intake among the participants.

\section{Conclusion}

For the diagnosis of acute pancreatitis, both serum lipase and gamma glutamyl transferase can be used. However, serum lipase is more diagnostic and more significantly increased than gamma glutamyl transferase, which was less sensitive and specific in patients with acute pancreatitis. Serum lipase could be used alone without any further estimation of other serum enzymes for the diagnosis of acute pancreatitis because of its high sensitivity and specificity in diagnosing this disease.

\section{Competing interests}

The author declares no competing interests. 


\section{References}

1. Toh SK, Phillips S, Johnson CD. A prospective audit against national standards of the presentation and management of acute pancreatitis in the South of England. GUT 2000; 46(2):39-43.

2. Goldacre M J, Roberts S E. Hospital admission for acute pancreatitis in an English population, 1963-98: database study of incidence and mortality. BMJ 2004; 328:1466-9.

3. Roberts SE, Akbari A, Thorne K, Atkinson M, Evans PA. The incidence of acute pancreatitis: impact of social deprivation, alcohol consumption, seasonal and demographic factors. Aliment Pharmacol Ther 2013; 38:539-48.

4. Shen HN, Lu CL, Li CY. Epidemiology of first-attack acute pancreatitis in Taiwan from 2000 through 2009: a nationwide populationbased study. Pancreas 2012; 41:696-702.

5. McKay CJ, Imrie CW. The continuing challenge of early mortality in acute pancreatitis. $\mathrm{Br} \mathrm{J}$ Surg 2004; 91:1243.

6. Munoz A, Katerndahl DA. Diagnosis and management of acute pancreatitis. Am Fam Physician 2000; 62:164-74.

7. Matull WR, Pereira SP, O'Donohue JW. Biochemical markers of acute pancreatitis. J Clin Pathol 2006; 59:340.

8. Yadav D, Agarwal N, Pitchumoni C S. A critical evaluation of laboratory tests in acute pancreatitis. Am J Gastroenterol 2002;971:309-18.

9. Forsmark CE, Baillie J. AGA Institute technical review on acute pancreatitis. Rev Gastroenterol Mex 2007; 72:257-85.

10. Swaroop V S, Chari S T, Clain J E. Acute severe pancreatitis. JAMA 2004; 291(23):28658.

11. Tenner S, Baillie J, DeWitt J, Vege SS, American College of Gastroenterology American College of Gastroenterology guideline: management of acute pancreatitis. Am J Gastroenterol 2013; 108:1400-15.

12. Batra H, Kumar A, Saha T, Misra P, Ambade V. Comparative study of serum amylase and lipase in acute pancreatitis patients. Indian Journal of Clinical Biochemistry 2015; 30(2):230-3.

13. Banks PA, Bollen TL, Dervenis C, Gooszen HG, Johnson CD, Sarr MG, et al. Acute Pancreatitis Classification Working Group. Classification of acute pancreatitis--2012 revision of the Atlanta classification and definitions by international consensus. Gut 2013; 62:102-11.

14. Lippi G, Valentino M, Cervellin G. Laboratory diagnosis of acute pancreatitis: in search of the Holy Grail. Crit Rev Clin Lab Sci 2012;49:18-31.

15. Frossard JL, Steer ML, Pastor CM. Acute pancreatitis. Lancet 2008; 371:143-52.

16. Werner J, Feuerbach S, Uhl W. Management of acute pancreatitis: from surgery to interventional intensive care. Gut 2005; 54(3):426-36.
17. UK working party on acute pancreatitis. UK guidelines for the management of acute pancreatitis. Gut 2005; 54(Suppl 3):iii1-9. 